\title{
The zinc-finger bearing xenogeneic silencer MucR in a- proteobacteria balances adaptation and regulatory integrity
}

\author{
Jian Jiao $\mathbb{B}^{1,2,3}$, Biliang Zhang ${ }^{1,2,3}$, Meng-Lin Li $\mathbb{D}^{1,2}$, Ziding Zhang $\mathbb{D}^{1 凶}$ and Chang-Fu Tian $\mathbb{D}^{1,2}$ \\ (c) The Author(s) 2021
}

\begin{abstract}
Foreign AT-rich genes drive bacterial adaptation to new niches while challenging the existing regulation network. Here we report that MucR, a conserved regulator in a-proteobacteria, balances adaptation and regulatory integrity in Sinorhizobium fredii, a facultative microsymbiont of legumes. Chromatin immunoprecipitation sequencing coupled with transcriptomic data reveal that average transcription levels of both target and non-target genes, under free-living and symbiotic conditions, increase with their conservation levels. Targets involved in environmental adaptation and symbiosis belong to genus or species core and can be repressed or activated by MucR in a condition-dependent manner, implying regulatory integrations. However, most targets are enriched in strain-specific genes of lower expression levels and higher AT\%. Within each conservation levels, targets have higher AT $\%$ and average transcription levels than non-target genes and can be further up-regulated in the mucR mutant. This is consistent with higher AT\% of spacers between -35 and -10 elements of promoters for target genes, which enhances transcription. The MucR recruitment level linearly increases with AT\% and the number of a flexible pattern (with periodic repeats of Ts) of target sequences. Collectively, MucR directly represses AT-rich foreign genes with predisposed high transcription potential while progressive erosions of its target sites facilitate regulatory integrations of foreign genes.
\end{abstract}

The ISME Journal (2022) 16:738-749; https://doi.org/10.1038/s41396-021-01118-2

\section{INTRODUCTION}

The distribution and abundance of organisms can be proximally explained in the scenario of the multi-dimensional niche while its ultimate explanation demands evolutionary understanding [1]. The match between organisms and niches is described by "adaptation" that involves past genetic changes in the scenario of Darwin's theory of evolution by natural selection [1]. The modern synthesis of evolution summarized by Julian Huxley in 1942 can be briefly outlined by its core tenet that "adaptive evolution" is a process, in which natural selection acts on heritable variability originating from accidental genetic changes, leading to increased frequency of advantageous variants and adaptation $[2,3]$. This adaptive evolution framework is still the root of the current standard evolutionary theory which recognizes four evolutionary processes (selection, mutation, recombination, and genetic drift) and only considers selection as a directional force increasing organismal adaptation [3]. In order to handle fluctuating abiotic and biotic stimuli, free-living bacteria and those pathogens or symbionts facultatively associated with eukaryotes are common to have an open pangenome which is characterized with a relatively small set of core genes and a large proportion of accessory genes [4]. Accessory genetic materials of bacteria are mainly provided by extensive horizontal transfer events within microbiota, which raises a challenging issue for maintaining many foreign "junk DNA" before the integration of few beneficial ones into existing transcriptional regulation network during adaptive evolution [5]. Indeed, it has been estimated that fine-tuned integration of a new gene into the regulation network of Escherichia coli can take millions of years [6]. Moreover, lineagespecific functions have been extensively recruited in bacterial adaptation [7]. Therefore, the maintenance and recruitment mechanism of foreign genes in the regulation scenario is a key to understand adaptation, speciation and the "home life" of living organisms, though it is largely unexplored.

New foreign DNA regions including both coding and noncoding sequences are usually AT-rich compared to the genome average [8]. Convergently evolved xenogeneic silencers preferring and maintaining AT-rich sequences [9] have been identified using chromatin immunoprecipitation (ChIP) and transcriptomic analysis for several model bacteria, such as H-NS in E. coli $[10,11]$ and Salmonella enterica [12, 13], MvaT in Pseudomonas aerugniosa [14], Lsr2 in Mycobacterium tuberculosis [15], and Rok in Bacillus subtilis [16], but it has not been explored how these xenogeneic silencers are involved in adaptive integration of foreign genetic materials into the regulation network (hereafter referred as "adaptive regulation"). With their temporally or spatially circumscribed effects, regulatory mutations in non-coding sequences rather than changes in coding region have been considered as a more common process during adaptive evolution [5, 17]. This is consistent with various examples in bacteria showing a contrasting turnover of target genes of the same transcription regulator in different species/strains, allowing their differential adaptation to distinct niches [18]. Consequently, we hypothesize that adaptive evolution in regulatory non-coding sequences targeted by

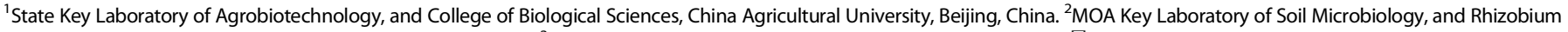
Research Center, China Agricultural University, Beijing, China. ${ }^{3}$ These authors contributed equally: Jian Jiao, Biliang Zhang. ${ }^{凶}$ email: zidingzhang@cau.edu.cn; cftian@cau.edu.cn 
xenogeneic silencers may underscore the recruitment of foreign genes during adaptation to various niches. This hypothesis of adaptive regulation involving both evolutionary and regulatory scenario can be considered as an updated version of the "ancestral" static transcriptional repression of foreign junk DNA by xenogeneic silencers.

To investigate the bacterial recruitment mechanisms for foreign genes within this adaptive regulation framework, we focused on rhizobia which live saprophytically in soil and form nitrogen-fixing bacteroids in legume root nodules in the nitrogen-depleted soil $[19,20]$. Recent coexpression network analysis of broad-hostrange Sinorhizobium fredii under free-living and symbiotic conditions demonstrated that the more conserved a gene is (from strain-specific to genus core genes), the greater its average transcriptional level and connection degree [21]. This pattern implies possible adaptive, not neutral, evolution of pangenome. This species and other Sinorhizobium members are also characterized by their multipartite genomes composed of chromosome, chromid, symbiosis plasmid and variable number of smaller plasmids [21, 22], among which chromid has plasmid-type maintenance and replication systems while having sequence signatures more similar to chromosomes than plasmids [23]. The MucR/Ros family protein has been intensively studied in rhizobia, particularly Sinorhizobium [24-32], showing pleiotropic roles under both free-living and symbiotic conditions. MucR/Ros homologs are conserved in a-proteobacteria and have a zinc-finger motif $[30,33]$. Recent studies demonstrate that MucR/Ros homologs show oligomerization and heat-stable features [34, 35], which are shared by the well-known xenogeneic silencer H-NS of E. coli [36]. Scattered evidence suggests that MucR homologs bind to AT rich regions in and around test bacterial promoters [9, 24, 26, 29, 37].

In this work, to investigate whether MucR is a global xenogeneic silencer and its potential role in adaptive regulation of foreign genes, ChIP-seq coupled with transcriptomic data were used to study S. fredii CCBAU45436 (SF45436) that is a microsymbiont of diverse legumes including cultivated and wild soybeans $[21,38,39]$. Adaptive regulation of MucR target genes belonging to individual pangenome subsets of different conservation levels (from genus core to strain-specific genes) or replicons (chromosome, chromid, symbiosis plasmid and two smaller accessory plasmids) were investigated in the wild-type and the mucR mutant under free-living and symbiotic conditions. The critical role of MucR in adaptive integration of foreign genes was discussed and highlighted in a working model in the context that AT-rich signature of foreign DNA can be progressively erased during adaptive evolution.

\section{MATERIALS AND METHODS}

\section{Bacterial strains, plasmids, primers and growth conditions}

$S$. fredii and $E$. coli strains, plasmids and primers used in this study are listed in Supplementary Table S1. S. fredii strains were grown at $28^{\circ} \mathrm{C}$ in TY or YEM medium [40]. The growth of $S$. fredii strains in YEM broth were monitored by Bioscreen C (Oy Growth Curves Ab, Finland). E. coli was cultured in Luria-Bertani medium at $37^{\circ} \mathrm{C}$. Concentrations of antibiotics were $30 \mu \mathrm{g} / \mathrm{mL}$ for nalidixic acid, $50 \mu \mathrm{g} / \mathrm{mL}$ for kanamycin, and $30 \mu \mathrm{g} / \mathrm{mL}$ for gentamicin.

\section{Comparative genomics and COG function annotation}

To define the pangenome subsets of SF45436, protein sequences from 12 Sinorhizobium genomes were clustered using the CD-HIT algorithm with a $70 \%$ sequence identity cut-off [41]. Hierarchical core/accessory genes were determined using the method described previously [21]. Briefly, four hierarchical subsets were defined as follows: Subset I (genus core of 12 Sinorhizobium strains), subset II (species core of 5 strains belonging to $S$. fredii excluding subset I), subset III (genes shared by three closely related $S$. fredii strains SF45436, SF25509 and HH103 but excluding subset I and II), and subset IV (SF45436-specific genes) [21]. COG annotation for SF45436 proteins was obtained using Reserved Position-Specific BLAST against COG database integrated in WebMGA [42].

\section{Chromatin immunoprecipitation sequencing (ChIP-seq)}

The plasmid pBGST-MucR1 with a PmucR1 promoting GST-MucR1 fusion protein was constructed based on pBBR1MCS-2 [43] and was conjugated into the mucR1 mutant, resulting the gstMucR1_pla strain used in subsequent experiments. For bacteroid isolation, $5 \mathrm{~g}$ of soybean nodules (30 dpi) were crushed within an ice-cold mortar containing $20 \mathrm{ml}$ of PBS added with $1 \%$ PVP (polyvinylpyrrolidone) and $1 \mathrm{~g}$ quartz sand. The homogenate was centrifuged at $4{ }^{\circ} \mathrm{C}, 400 \times \mathrm{g}$ for $10 \mathrm{~min}$ to remove quartz sand and plant tissue, and the supernatant was further centrifuged at $4{ }^{\circ} \mathrm{C}$, $10,000 \times \mathrm{g}$ for $5 \mathrm{~min}$ to pellet bacteroid cells. Bacteroids or TY cultures $\left(\mathrm{OD}_{600}=1.2\right)$ were resuspended in PBS $(\mathrm{pH} 7.4)$, and formaldehyde was added to a final concentration of $1 \%$. Crosslinking reactions were sustained for $15 \mathrm{~min}$ at room temperature with gentle shaking, and stopped by adding glycine to a final concentration of $0.125 \mathrm{M}$ for $5 \mathrm{~min}$. Crosslinked cells were ground into fine powder, resuspended with ChIP buffer $(50 \mathrm{mM}$ Tris- $\mathrm{HCl}$ (pH8.1), $150 \mathrm{mM} \mathrm{NaCl}, 5 \mathrm{mM}$ EDTA, 1\% Triton X-100, 0.1\% sodium deoxycholate) plus protease inhibitor cocktail (CWbiotech). Cell lysates were sonicated on ice to shear DNA fragments to an average length of $300 \sim 400 \mathrm{bp}$ and cleared by centrifugation at $14,000 \times \mathrm{g}$ for $15 \mathrm{~min}$ at $4{ }^{\circ} \mathrm{C}$ Supernatants were normalized by protein content and pre-cleared with 40 $\mu \mathrm{l}$ proteinA/G agarose (CWbiotech). $10 \%$ of the supernatant was removed and used for total chromatin input DNA preperation. For each ChIP reaction, $5 \mu \mathrm{l}$ monoclonal anti-GST (CWbiotech) was added and incubated overnight at $4{ }^{\circ} \mathrm{C}$ with $40 \mu \mathrm{l}$ of protein-A/G agarose beads pre-saturated with BSA. The beads were washed twice with ChIP buffer, once with high salt buffer (50 mM Tris- $\mathrm{HCl}$ (pH8.1), $500 \mathrm{mM} \mathrm{NaCl}, 5 \mathrm{mM}$ EDTA, 1\% Triton X$100,0.1 \%$ sodium deoxycholate) and $\mathrm{LiCl}$ buffer $(10 \mathrm{mM}$ Tris- $\mathrm{HCl}(\mathrm{pH} 8.1)$, $25 \mathrm{mM} \mathrm{LiCl}, 0.5 \%$ sodium deoxycholate, $1 \mathrm{mM}$ EDTA), and twice with TE buffer ( $10 \mathrm{mM}$ Tris-HCl (pH 8.1) and $1 \mathrm{mM}$ EDTA). Protein-DNA complexes were eluted in $500 \mu$ l elution buffer ( $\left.1 \% \mathrm{SDS}, 0.1 \mathrm{M} \mathrm{NaHCO}_{3}\right)$, supplemented with $\mathrm{NaCl}$ to a final concentration of $300 \mathrm{mM}$ and incubated overnight at $65^{\circ} \mathrm{C}$. Samples were treated with $0.5 \mathrm{mg}$ of Proteinase $\mathrm{K}$ for $2 \mathrm{~h}$ at $45^{\circ} \mathrm{C}$. DNA was extracted using phenol:chloroform:isoamyl:alcohol (25:24:1), ethanol precipitated using Dr. GenTLE Precipitation Carrier (TAKARA). Library construction (300-400 bp) and deep sequencing (paired-end 125 using HiSeq 2500; Illumina) were performed by SinoGenoMax-Beijing, with the total chromatin input DNA as control. Three biological replicates were tested.

To investigate the potential side effects of plasmid borne GST-MucR1 in the gstMucR1_pla strain, a GST-MucR1 in situ expression strain, gstMucR1_chr, was constructed through homologous recombination by using pJQGST-MucR1 derived from pJQ200SK [44] carrying up- and downstream homologous fragments of mucR1 (Supplementary Table S1). Western blotting was carried out to analyze the specificity of anti-GST monoclonal antibody and to determine the accumulation of GST-MucR1 protein in gstMucR1_pla and gstMucR1_chr. These two strains were further compared with the wild type for their growth in YEM broth and plate, and symbiotic performance on host plants as previously described [21]. To verify the ChIP-seq data, seven representative peaks with different enrichment fold change values were tested by ChIP-qPCR with diluted DNA recovered from input and ChIP samples as templates. qPCR was performed by using QuantStudioTM 6 Flex and 2x RealStar Green Mixture (Genstar). ZnuA coding sequence [45] was used as the reference for normalization of MucR1 recruitment levels. Three independent biological replicates were analyzed.

\section{High-throughput data analysis}

Clean ChIP-seq reads were mapped to the genome using bowtie2 to generate BAM files which were used for peak calling by running MACS2 (version 2.1.0) [46]. The parameters for peak and summit (peak center) calling are as follows: "macs2 callpeak --call-summits -B -g 6913799 --nomodel --extsize 100 --bw 300 --qvalue 0.01 --keep-dup 1 -f BAMPE --outdir MACS2_es100_bw300_BAMPE -t ChIP.bam -c Input.bam -n ChIPInput", in which "bw" and "extsize" represent bandwidth (half of the estimated sonication size, bp) and extension size (the minimum length of peaks, bp), respectively. Positive peaks $(F D R<0.01)$ with their summit distance less than $200 \mathrm{bp}$ for different samples were re-allocated with the same peakID. A gene associated with a peak summit in its regulation region ( $-500 \mathrm{bp}$ to $+100 \mathrm{bp}$ around the start codon) was assigned as target genes directly regulated by MucR1. Circos visualization of high- 
throughput data was accomplished by using ShinyCircos package in R [47]. When sequence features of peaks were analyzed, the central region of 200 bp (with summit in the peak center) was used.

Over-represented motif of ChIP-seq peaks was determined by analyzing the 200 bp central region using the web-based MEME-ChIP module. One of "class A flexible patterns" $\Pi x x x G x x x T x x x x x x x x x x T$ [48] highly coincides with the DNA-binding site resolved in a Ros87-DNA docking model [49] and was scanned for its distribution in individual replicons and genomic islands of SF45436. Motifs with no more than one insertion or deletion in the first two non-conserved regions and no more than two insertions or deletions in the third non-conserved region were recorded. Genomic islands were identified by using IslandViewer 4 with three different prediction methods: IslandPick, IslandPath-DIMOB, SIGI-HMM [50]. To summarize the distribution of this flexible pattern in SF45436 genome, the distance between the middle position of the motif and the first nucleotide of gene was used. This allowed us to get a general picture on the relative abundance of the flexible pattern along the promoter (boundary: $-500 \mathrm{bp}$ ) and coding regions of genes. Since not all genes are the same length, the boundary for counting motifs within coding regions was set as $+2000 \mathrm{bp}$. For those genes shorter than $2000 \mathrm{bp}$, their downstream intergenic regions were not included in the analysis to avoid interfering with true intragenic signals. Similarly, the distance between the summit position of ChIP-seq peak and the first nucleotide of gene was calculated to determine the relative abundance of ChIP-seq summits in the region from $-500 \mathrm{bp}$ to $+100 \mathrm{bp}$. Kernel density (probability density) plots were then produced for visualizing these abundance data of the flexible pattern and ChIP-seq summits by using R package ggplot2 [51]. The number of class $A$ flexible patterns and AT content were also calculated for the $200 \mathrm{bp}$ central region of ChIP-seq peaks.

Oligonucleotide usage deviation values were calculated as described earlier [52]. Briefly, the zero-order Markov method is designed to determine the expected number of oligonucleotides by removing biases in mononucleotide frequencies. The normalized oligonucleotide deviation value for a word $\mathrm{W}$ is calculated by dividing the observed counts by the expected counts. MucR1 binding preference on each oligonucleotide was evaluated by Spearman's rho between oligonucleotide usage deviations in the $200 \mathrm{bp}$ central regions of ChIP-seq peaks and MucR1 recruitment levels.

Promoter sequences of Sinorhizobium identified previously [53] were used as input for the prediction of promoters identified by sigma factors $\mathrm{RpoD}$, RpoH1, RpoH2, RpoE2, and RpoN in SF45436. The -35 and -10 elements of input promoters were extracted to construct separate positionspecific scoring matrix. Profiles searching were performed on the SF45436 genome for putative promoter elements using PoSSuMsearch [54]. After profile searching, those putative promoters with the spacer length between -35 and -10 elements does not comply with the spacer length threshold were removed [53]. AT content of spacer sequences within the predicted intergenic and intragenic promoters was then calculated.

\section{Protein purification}

The plasmids overexpressing His6-SUMO-tagged MucR1/MucR2 were constructed by cloning mucR1/mucR2 into pET30a-SUMO. Soluble His $s_{6}-\mathrm{SUMO}$, $\mathrm{His}_{6}-\mathrm{SUMO}-\mathrm{MucR} 1$ and $\mathrm{His}_{6}-\mathrm{SUMO}-\mathrm{MuCR} 2$ were purified from E. coli BL21 (DE3) containing pHis-MucR1 and pHis-MucR2, respectively, under native conditions. Briefly, $500 \mathrm{ml}$ culture was treated with $0.2 \mathrm{mM}$ IPTG at $16^{\circ} \mathrm{C}$ overnight. Then the cells were collected, resuspended in lysis buffer $(25 \mathrm{mM}$ Tris- $\mathrm{HCl}, \mathrm{pH}$ 8.0, $250 \mathrm{mM} \mathrm{NaCl}, 50 \mathrm{mM}$ imidazole, $5 \%$ glycerol) and lysed by ultrasonic shear until the suspension turned to be clear. After centrifugation at $250,000 \times \mathrm{g}$ the supernatant was loaded onto a chromatography column filled with $1 \mathrm{ml}$ Ni-nitrilotriacetic acid resin (Ni-NTA, Qiagen). After washing with the same buffer of 5-10 fold volumes, the recombinant proteins were eluted using the elution buffer (lysis buffer supplemented with $500 \mathrm{mM}$ imidazole) with a linear gradient. The elution fractions were purified by sizeexclusion chromatography using a Superdex 200 10/30 column (GE Healthcare) and the SEC buffer (20 mM Tris-HCl, pH 8.0, $250 \mathrm{mM} \mathrm{NaCl}, 5 \%$ glycerol) and were divided into aliquots and stored at $-80^{\circ} \mathrm{C}$.

\section{Electrophoretic mobility shift assays (EMSA)}

The $250 \mathrm{bp}$ promoter region of vis $N$ covering MucR1-binding sites was amplified in a PCR. The $5^{\prime}$-Cy5-labeled PCR fragments were purified and used as probes. The EMSAs were performed as follows: for each reaction, $12.3 \mathrm{nM}$ Cy5-labeled DNA probe and various concentrations of test proteins were diluted into the binding buffer $(25 \mathrm{mM}$ Tris (pH7.5), 100 $\mathrm{mM} \mathrm{KCl}, 5 \mathrm{mM} \mathrm{MgCl}$, $5 \%$ glycerol and $0.05 \%$ dodecyl maltoside, $0.5 \mathrm{mg} /$ $\mathrm{mL} \mathrm{BSA}, 0.1 \mathrm{mg} / \mathrm{mL}$ sonicated salmon sperm DNA) in a final volume of 10 $\mu \mathrm{l}$. The samples were incubated at room temperature for 20-30 $\mathrm{min}$, then separated on a $6 \%$ TB polyacrylamide gel (no EDTA) and the gel was scanned with a Typhoon FLA 9000 imager (GE Healthcare).

\section{RESULTS AND DISCUSSION}

\section{ChIP-seq uncovers MucR1 as a global DNA-binding protein}

We have demonstrated that MucR1 of SF45436, rather than its paralog MucR2 carrying a frameshift mutation, is essential in forming mucoid colonies and nitrogen-fixing nodules on soybean plants [31]. MucR1 in SF45436 has the conserved prokaryotic zinc-finger motif $\mathrm{X}_{2}$ Cys- $\mathrm{X}_{2}$-Cys- $\mathrm{X}_{9}-\mathrm{His}_{2} \mathrm{X}_{3}-\mathrm{His}_{2} \mathrm{X}_{2}$ (Fig. 1A) that is a characterized feature of ROS_MUCR (PF05443) family protein widely distributed in aproteobacteria (Fig. 1B). To determine the direct targets of MucR1 in SF45436 genome, a GST-MucR1 expressing strain driven by the promoter of mucR1 was constructed in the background of the $\triangle m u c R 1$ mutant. Similar to the wild-type SF45436, the resulting strain formed mucoid colonies and nitrogen-fixing nodules on soybean plants (Fig. 1C), indicating that the N-terminal fused GST-tag had no significant influence on the function of MucR1. Western blot using both free-living cells and symbiotic bacteroids demonstrated the specificity of monoclonal anti-GST antibody (Fig. 1D) that was used in subsequent ChIP-seq experiment.

Previous RNA-seq analysis revealed MucR1 as a pleiotropic regulator under both free-living ( $T Y$ medium, $\mathrm{OD}_{600}=1.2$ ) and symbiotic conditions (nodules from cultivated soybean JD17, 30 days post inoculation [DPI]) [31]. In this work, ChIP-seq was performed for both free-living cells (FC; TY medium, $\mathrm{OD}_{600}=1.2$ ) and bacteroid cells (BC; nodules from cultivated soybean JD17, 30 DPI) under the same conditions. Representative ChIP-seq peaks observed in a gene cluster involved in flagellum-dependent motility and chemotaxis are shown in Fig. 2A. Electrophoretic mobility shift assay verified that MucR1 can directly bind its target sequences such as the promoter region of vis $N$ (Fig. 2B) while MucR2 carrying a frameshift mutation cannot. $84 \%$ (1154) of FC peaks and $85.9 \%$ (806) of BC peaks were reproducibly uncovered in all three biological replicates, and $91.6 \%$ (1258) of FC peaks and $94.5 \%$ (886) of $B C$ peaks were observed in at least two biological replicates (Supplementary Fig. S1A; $q$-value $<0.01$ ). When the data from three biological replicates were combined, 1484 and 1068 significant peaks were identified $(q$-value $<0.01)$ in FC and BC samples, respectively (Fig. $2 \mathrm{C}$ and Supplementary Table S2). These peaks constitute a set of 1551 unique ChIP-seq peaks, of which 1002 were detected under both free-living and symbiotic conditions (Supplementary Table S2). Notably, there is a strong positive correlation between the enrichment fold values of shared ChIP-seq peaks under two conditions (Supplementary Fig. S1B; Pearson's rho = $0.90, P$ value $=2.2 \mathrm{e}-16$ ) whereas the enrichment fold values of condition-specific ChIP-seq peaks are generally low (531/549 below 5), such as those shown in Fig. 2A. This phenomenon is well presented in a Circos overview of ChIP-seq mapping patterns which are indistinguishable between FC-peaks and BC-peaks (Fig. 2C). These results indicate that the recruitment levels of MucR1 to most of its targets are not altered by two contrasting conditions, though targets specific to either free-living cells or bacteroids can also be detected implying potential condition-dependent regulation. This is in line with the pleiotropic role of MucR homologs in environmental adaptation of a-proteobacteria $[31,37,55]$ and condition-modulated interactions of xenogeneic silencers with DNA [56].

By associating the peaks with nearby genes, totally 1350 protein encoding genes were defined as direct MucR1 target genes. Within these target genes, 1307 and 911 genes were found in FC and BC ChIP-seq experiments, respectively, and 868 genes are shared by two conditions (Supplementary Table S2). Noteworthy, these numbers of direct target genes are very conservative, considering that genes downstream of the first gene within a polycistronic operon were not included (such as those polycistrons in Fig. 2A).

To test if there was potential influence on the identification of ChIPseq peaks caused by the expression level of plasmid borne GST fusion 
A

\begin{tabular}{ll}
\cline { 2 - 2 } Eukaryotes & $\mathrm{X}_{2}$-Cys- $\mathrm{X}_{2-4}-$ Cys- $\mathrm{X}_{9 / 12}-\mathrm{His}-\mathrm{X}_{3-5}-\mathrm{His}-\mathrm{X}_{2}$ \\
Prokaryotes & $\mathrm{X}_{2}$-Cys- $\mathrm{X}_{2}-$ Cys- $\mathrm{X}_{9}-$ His- $\mathrm{X}_{3}$-His- $\mathrm{X}_{2}$
\end{tabular}

MucR1 of SF45436

MSENTLGTSNELLVELTAEIVAAYVSNHVVPVAELPTLIADVHSALNNTTAPAPV IVPVEKPKPAVSVRKSVQDDQIICLECGGTFKSLKRHLMTHHNLSPEEYREKW DLPADYPMVAPAYAEARSRLAKEMGLGQRRKRRGK

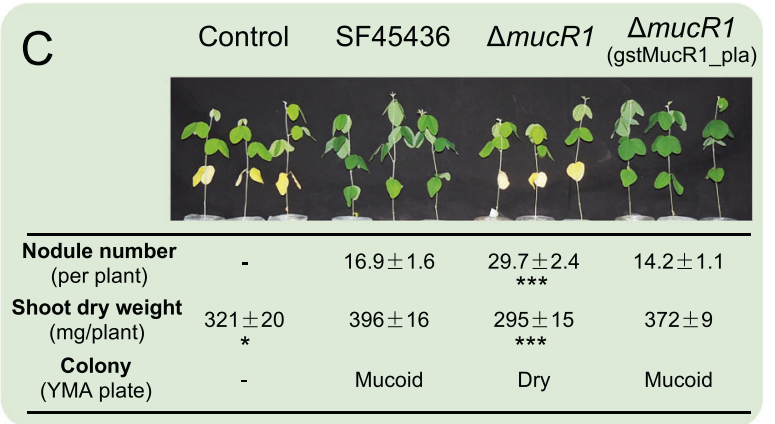

D

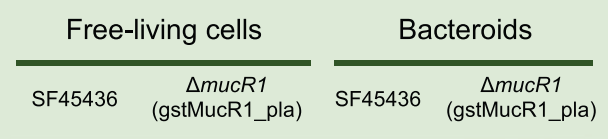

anti-GST

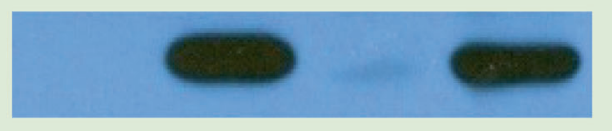

Fig. 1 The zinc-finger regulator MucR1 in soybean microsymbiont SF45436. A MucR1 contains a conserved C2H2 zinc-finger motif. B The neighbor-joining phylogenetic tree of MucR/Ros homologs of the ROS_MUCR (PF05443) family proteins showing their enrichment in $\alpha$-Proteobacteria. 273 Pfam seed sequences and 41 sequences from representative lineages not well covered by seed sequences were analyzed. The number of sequences from strains of corresponding phylum/class/order is shown in brackets. C The symbiotic and free-living defects of $\Delta$ mucR1 can be recovered by introducing PBGST-MucR1. The PmucR1 was used to drive the expression of GST-MucR1. Average values and standard error of the mean are shown. Significant difference between the mean of each treatment and that of SF45436 is indicated ( $t$ test; ${ }^{*}, P$ value $<0.05 ;{ }^{* * *}, P$ value $<0.001$; more than eight plants were scored). D Detection of GST-MucR1 in free-living cells and bacteroids from soybean nodules by Western blot using anti-GST antibody.

MucR (gstMucR1_pla), a derivative carrying a chromosomal borne GST fusion MucR (gstMucR1_chr) was constructed. Despite a slightly higher expression level of GST fusion MucR in the gstMucR1_pla strain compared to the gstMucR1_chr strain (Supplementary Fig. S2), seven representative ChIP-seq peaks of various enrichment levels identified in the gstMucR1_pla strain were also identified in the gstMucR1_chr strain in ChIP-qPCR (Supplementary Fig. S2). Moreover, the free-living and symbiotic phenotypes (Supplementary Fig. S2 and Supplementary Table S3) are similar between two strains. Therefore, the ChIP-seq results obtained in the gstMucR1_pla are reproducible.

\section{MucR1 directly regulates AT-rich core genes involved in stress and symbiosis adaptation}

MucR1 negatively regulates several key functions involved in stress and symbiosis adaptation (Fig. 3). For example, rpoE5 encodes a sigma factor responding to general stress; $[57,58]$ visNR, rem and several gene clusters encode transcriptional factors or functional components involved in motility $(\mathrm{fla} / \mathrm{flg} / \mathrm{fli} / \mathrm{mot})$, chemotaxis $(\mathrm{mcp} / \mathrm{che})$ and pilus assembly $(c p a) ;[59,60]$ nodD2 encodes a negative regulator of nodulation genes in $S$. fredii; [61, 62] a gene cluster encodes structure components of T3SS (type three secretion system) and the effector NopP modulating compatibility of $S$. fredii with legume hosts [63-65]. On the other hand, there are few processes subject to positive regulation by MucR1 (Fig. 3), such as exo genes involved in the biosynthesis of succinoglycan exopolysaccharide [24], phoUB regulating phosphate starvation machinery [66,67], and rirA coding an iron responsive regulator required for effective symbiotic nitrogen fixation of $S$. fredii on soybean plants [68]. Among few studies of MucR-DNA interactions, evidence for direct transcriptional activation role of MucR is so far just available for exoY encoding a galactosyltransferase that initiates the assembly of repeating unit of succinoglycan exopolysaccharide [24]. These well-characterized functional target genes have higher AT than the average of individual pangenome subsets and most of them belong to genus or species core genes (Fig. 3; blue circles with numbers 1 and 2). These findings suggest MucR1 as a master regulator for these AT-rich core genes providing adaptation benefits under either free-living or symbiotic conditions, indicating successful regulatory integrations. For example, a MucR-repressed cryptic gene cluster directing exopolysaccharide production can be activated by a local regulator CuxR in the presence of c-di-GMP in Sinorhizobium meliloti $[32,69]$.

\section{MucR1 target genes are enriched in less conserved strain- specific foreign genes}

It is noteworthy that MucR1 targets are more intensively distributed among accessory genes of $S$. fredii (the third ring in Fig. $2 \mathrm{C}$, gene content excluding species core genes) and plasmids or genomic regions with GC\% lower than the average (the fourth ring in Fig. $2 \mathrm{C}$ ). These global pictures are statistically verified in Fig. 4. AT\% of target genes is generally higher than that of non-target genes ( $t$-test, $P$ values $<0.001$ ) within individual replicons (chromosome, chromid pSFb, symbiosis plasmid pSFa, and two accessory plasmids pSFd/ pSFe; Fig. 4A) or core/accessory subsets of different conservation levels ranging from genus core (Subset I) to SF45436-specific genes (Subset IV) (Fig. 4B). As high AT\% is a characterized feature of foreign genes in Proteobacteria [70], these results imply MucR1 as a global regulator associated with foreign genes on different replicons and across different conservation levels.

This view is in line with a significant enrichment of MucR1 target genes on chromid (pSFb; Fisher's exact test, $P$ value $<0.05$ ), symbiosis plasmid (pSFa; Fisher's exact test, $P$ value $<0.001$ ) and an accessory plasmid pSFe (Fisher's exact test, $P$ value $<0.01$ ) whereas depletion on chromosome (Fisher's exact test, $P$ value $<0.001$ ) (Fig. $4 C$ ). On the other hand, targets are significantly enriched in the less conserved 

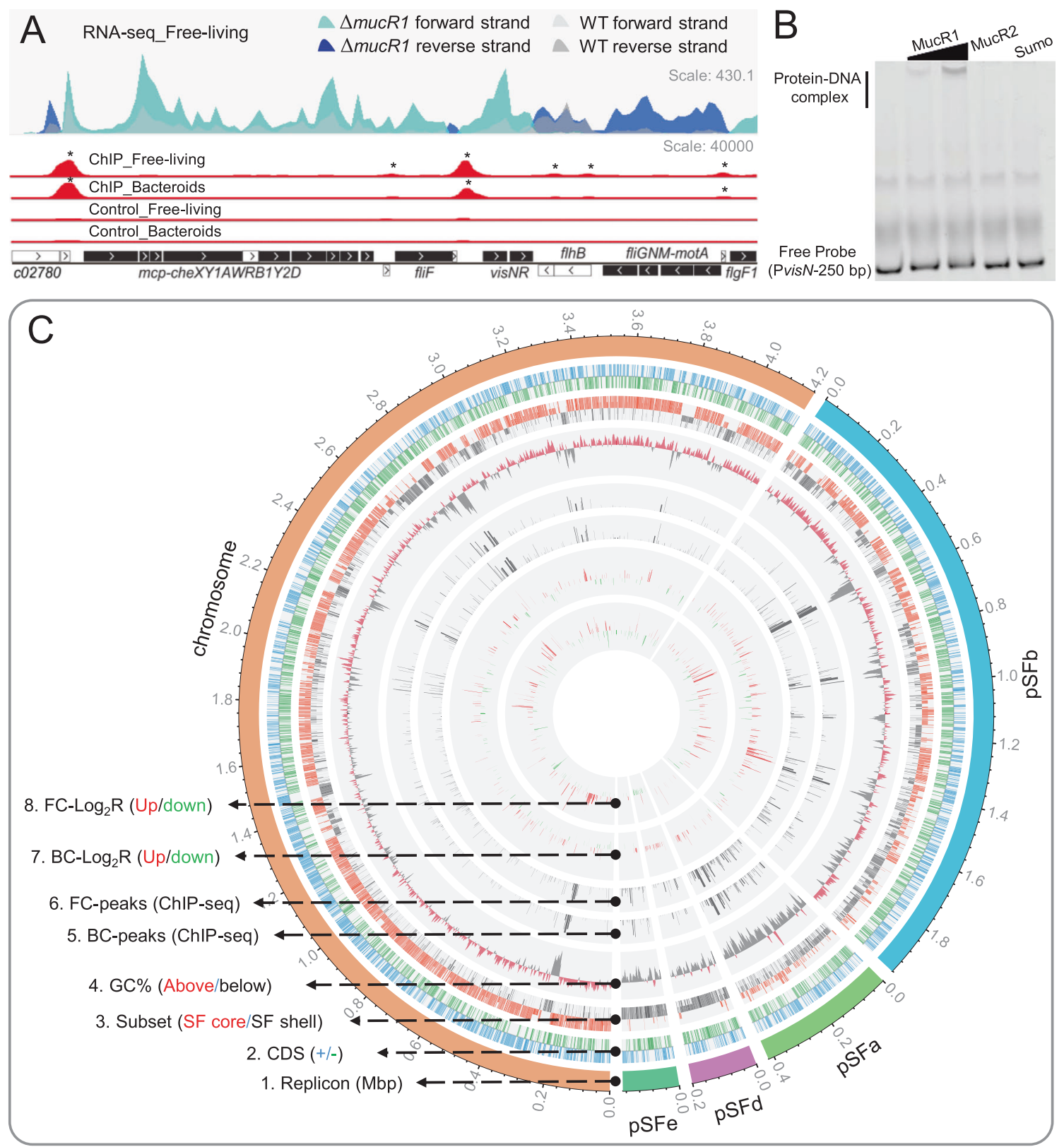

Fig. 2 Mapping ChIP-seq and RNA-seq reads on the multipartite genome. A Representative figure showing coverage of reads from ChIP-seq (both free-living cells collected at $\mathrm{OD}_{600}=1.2$ in TY medium and bacteroids) on genetic loci involved in flagellum-dependent motility and chemotaxis. MucR1 ChIP-seq peaks identified are indicated by asterisks (Enrichment fold $>1$, FDR $<0.001$ ). Coverage of reads from strandspecific RNA-seq (free-living cells collected at $\mathrm{OD}_{600}=1.2$ in TY medium) is also shown. DEGs between SF45436 and the mucR1 mutant are indicated as black box $\left(\log _{2}\right.$ Ratio $(m u c R 1 / S F 45436)>1.0$, FDR $\left.<0.001\right)$. B Electrophoretic mobility shift assay showing the direct binding of His ${ }_{6}{ }^{-}$ SUMO-MucR1 (13.5 and $27 \mu \mathrm{M})$ on the promoter region of vis $N$ in vitro. His ${ }_{6}$ SUMO-MucR2 (27 $\mu$ M) and His6-SUMO (27 $\mu$ M) were negative controls. C Circos overview of the genome information and ChIP-seq/RNA-seq mapping results. 1. Replicons in different colors. 2. CDS on the "+" strand and "-" strand in blue and green color, respectively. 3. Genes within four hierarchically divided core/accessory subsets represented by different bars: Subset I (genus core; red/long), subset II (S. fredii core excluding subset I; red/short), subset III (genes shared by SF45436, SF25509 and HH103 but excluding subset I and Il; black/short), subset IV (SF45436-specific genes; black/long). 4. GC\% with window size of 1 kb with red and black color showing the value above and below the whole genome average, respectively. 5-8. BCs-peaks (bacteroids), FCs-peaks (free-living cells), $\log _{2} \operatorname{Ratio}(\Delta$ mucR1/SF45436) in bacteroids and the free-living state are sequentially shown from the 5 th to the 8 th rings. Upand down-regulated genes are indicated by red and green color on the 7th and 8th rings.

strain-specific Subset IV (Fisher's exact test, $P$ value $<0.001$ ) whereas depleted in the more conserved genus core Subset I (Fisher's exact test, $P$ value $<0.001)$. Moreover, peaks with higher MucR1 recruitment levels were more frequently found on plasmids (Supplementary Fig. S3A) and associated with genes belonging to the less conserved subset IV (Supplementary Fig. S3B). In short, these findings suggest a preference of MucR1 to less conserved strainspecific foreign genes of higher AT content.

MucR1 down regulates its AT-rich targets with predisposed high transcription potential

ATP and UTP are characterized by lower energy cost and more abundant over GTP and CTP [71], and there is experimental 


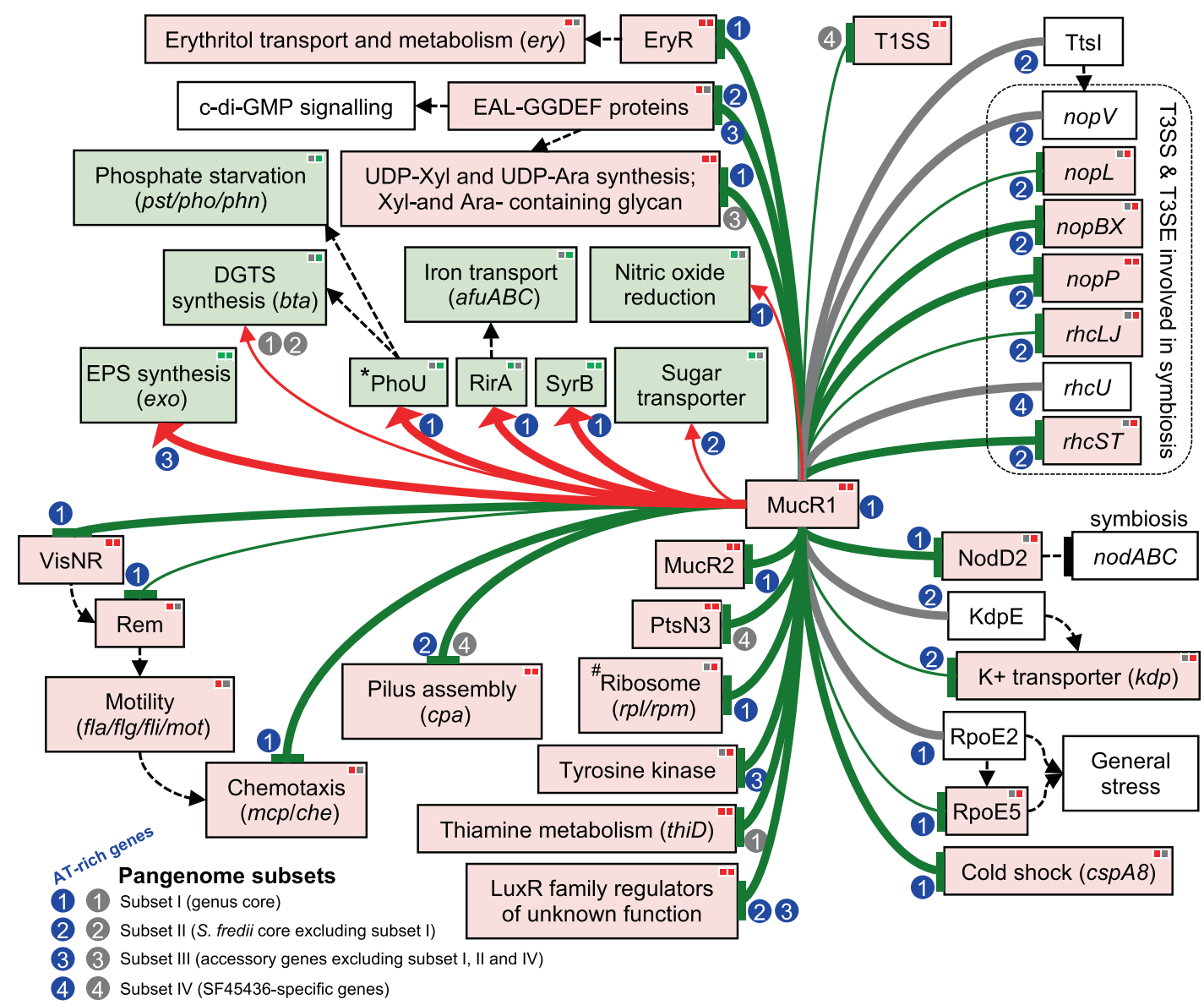

Fig. 3 MucR1 directly regulates AT-rich functional genes of different conservation levels. Summary of MucR1 target genes and related expression profiles. Red and green solid lines indicate activation and repression, respectively, by MucR1. Thick and thin solid lines represent peaks enriched by the fold above 5 and the fold between 1 and 5, respectively. MucR1-dependent expression is indicated by the background color of boxes: pink (up-regulated in the mucR1 mutant) and light green (down-regulated in the mucR1 mutant). Arrows and "T" shape lines in black color show the activation and repression effects documented in literatures. DEGs detected in different conditions are indicated by different colors (red, $\Delta m u c R 1 / W T$, up-regulated; green, $\Delta m u c R 1 / W T$ down-regulated; left, free-living cells in TY medium at OD600 =1.2; right, JD17 bacteroids). *, a strong recruitment signal was detected within ORF of $p s t B$ which is located in the pstSCAB-phoUB operon. \#, differentially expressed genes were $c 11230$ and c11240 encoding ribosomal proteins L16p and L29p. Numbers in circles indicate four pangenome subsets of different conservation levels, and blue circles indicate that these genes are AT-rich in corresponding pangenome subsets.
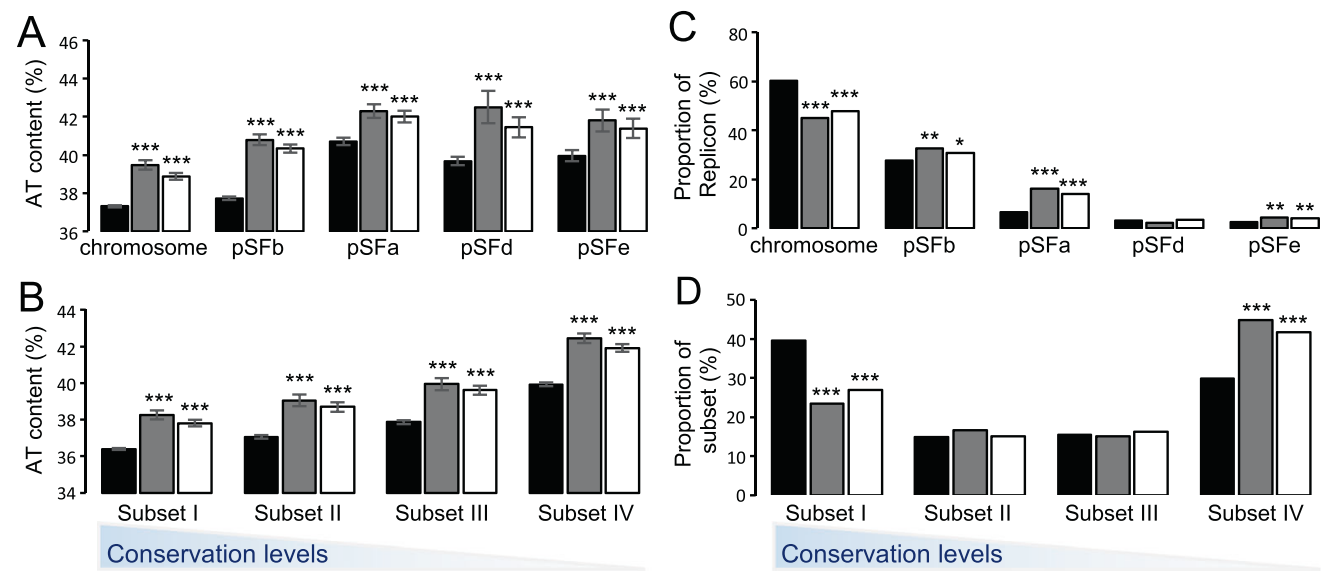

All genes Target genes_bacteroids $\square$ Target genes_free-living

Fig. 4 MucR1 targets across subsets of different conservation levels and replicons. AT\% content of peak-associated genes within individual replicons (A) and different core/accessory subsets (B). Error bars represent standard error of the mean. Significant difference between target AT\% and the corresponding average value (black column) of the same subset or replicon is indicated ${ }^{*}, P$ value $<0.05 ;{ }^{*}, P$ value $<0.01 ;{ }^{* *}, P$ value $<0.001 ; t$ test). Enrichment analysis of MucR1-target genes for each replicons (C) and core/accessory subsets (D). Subset I (genus core), subset II (S. fredii core excluding subset I), subset III (genes shared by three closely related strains SF45436, SF25509 and HH103 but excluding subset I and II), subset IV (SF45436-specific genes). Significant enrichment/depletion is indicated $\left({ }^{*}, P\right.$ value $<0.05 ;{ }^{* *}, P$ value $<0.01 ;{ }^{* * *}, P$ value $<0.001 ;$ Fisher's exact test). 

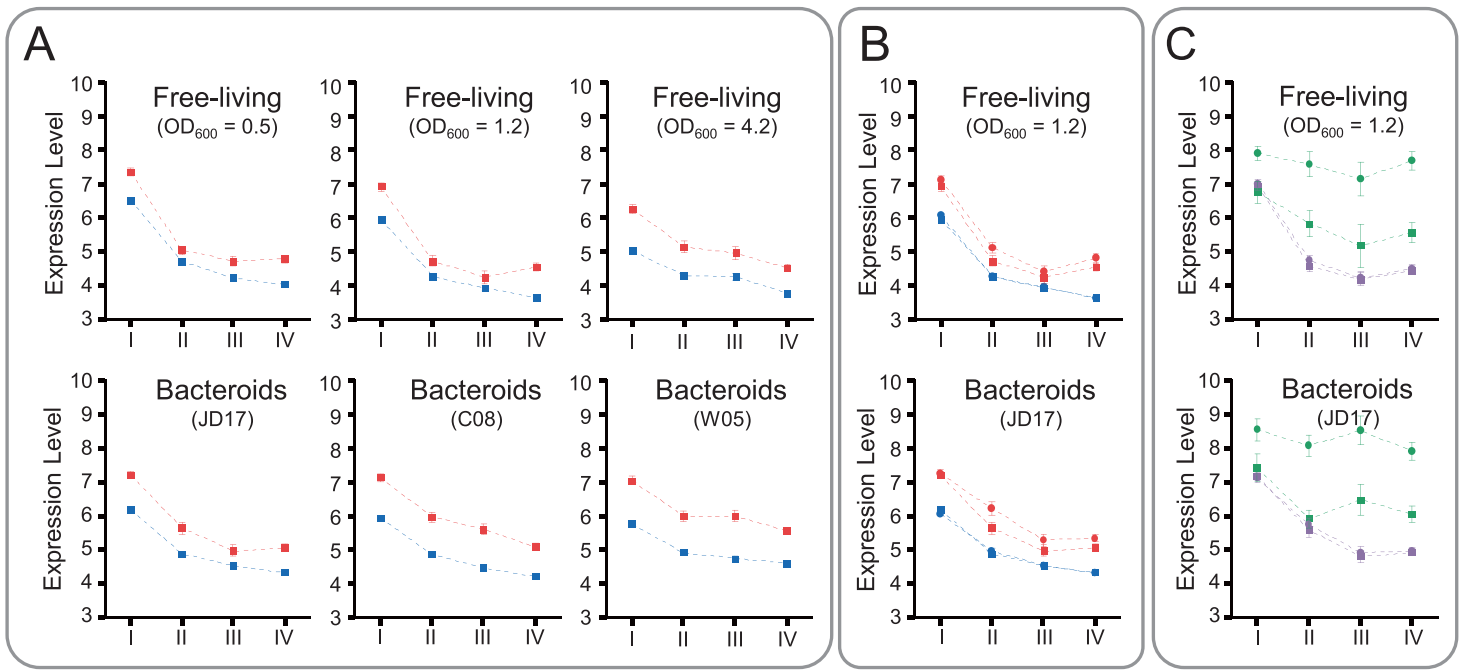

$$
\text { --1- WT_target }
$$

- $\triangle m u c R 1$ target

- WT_target_nonDEG

$\rightarrow \triangle$ mucR1_target_nonDEG

- WT_nontarget

- $\triangle m u c R 1$ nontarget

-1- WT_target_DEG

$\rightarrow \Delta m u c R 1$ target_DEG

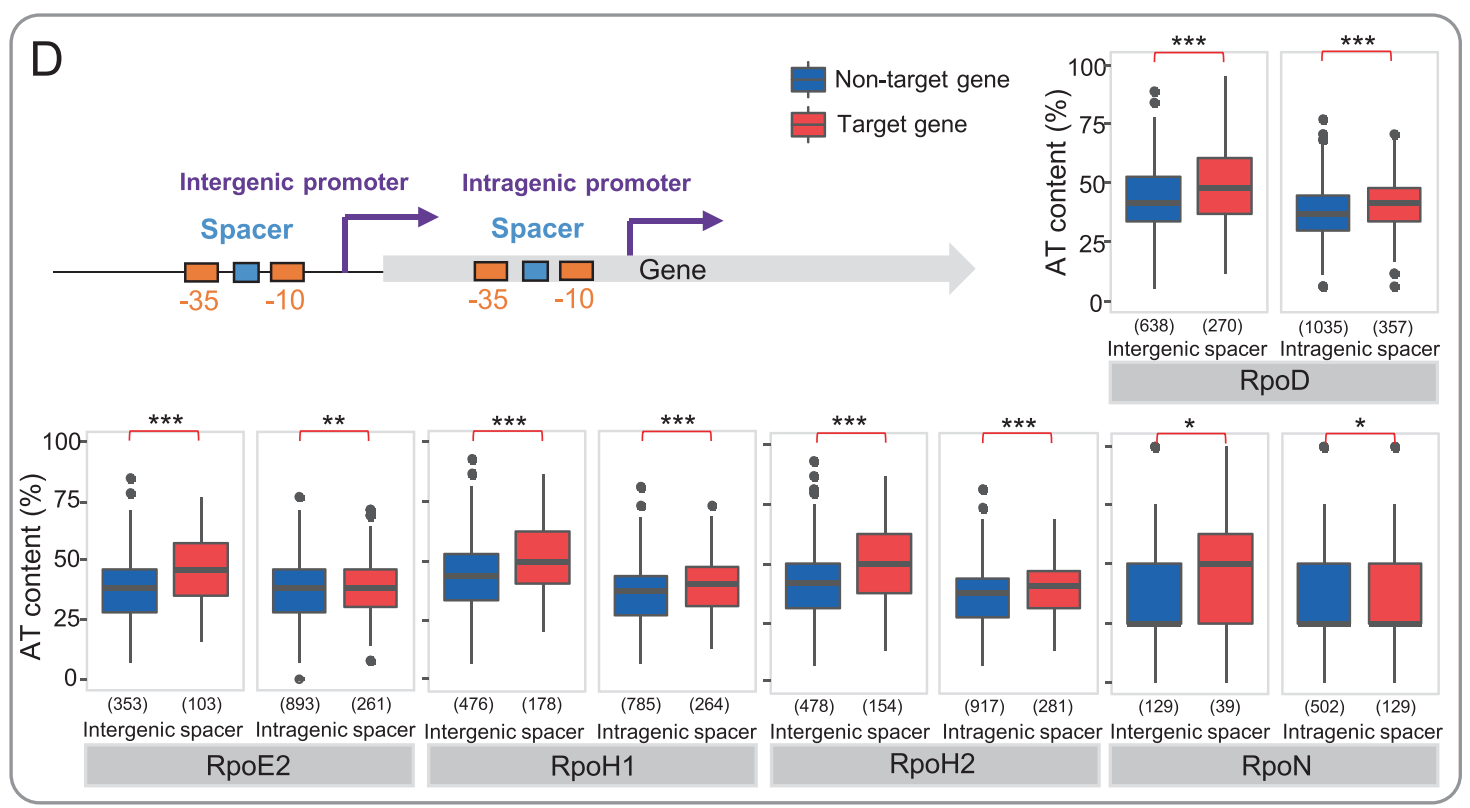

Fig. 5 MucR1 down regulates its highly transcribed target genes across conservation levels. A MucR1 target genes were generally transcribed at a higher level than non-target genes under both free-living (TY medium; $\mathrm{OD}_{600}=0.5,1.2$ and 4.2 ) and symbiotic conditions (Bacteroids from nodules of cultivated soybeans JD17 and C08, and wild soybean W05). B Average transcription level of MucR1 target genes was higher in the mucR1 mutant than in SF45436. C A subset of MucR1 target genes were differentially expressed in a condition-dependent manner. A-C I-IV along the horizontal axis represent pangenome subsets of different conservation levels as defined in Figs. 2-4. D AT\% of spacer sequences between -10 and -35 elements of predicted intergenic and intragenic promoters of different sigma factors. The numbers of spacers identified and analyzed for target and non-target genes are shown in brackets. ${ }^{*}, P$ values $<0.05 ;{ }^{*}, P$ values $<0.01 ;{ }^{* *}, P$ values $<$ 0.001 (Student's $t$ test).

evidence suggesting that cell-level selection drives the genomes of extrachromosomal replicons toward higher A + T contents [72]. There is a pervasive mutational bias from GC toward AT in diverse bacteria [73]. However, an earlier test of potential fitness effects of AT content showed that, independent of adaptive codon use, artificial induction of AT-rich mRNA synonymous variants of two non-native genes (GFP and a phage DNA polymerase) in E. coli led to lower growth rates compared to induction of GC-rich variants [74]. Therefore, AT-rich genes seem to be better candidates to be banked in bacterial pangenome, but should be tightly regulated.

To further investigate transcriptional characteristics of MucR1 target genes, we analyzed RNA-seq data from SF45436 or its mucR1 mutant samples collected under various free-living and symbiotic conditions [21, 31]. Average transcriptional levels of both target and non-target genes decrease with the conservation levels from genus core to strain-specific genes (Fig. 5A), while AT content increases from genus core to strain-specific genes (Fig. 4B). Therefore, newer pangenome members of higher AT content are more likely down-regulated before successful integration with the existing regulatory network. This is supported by a genome scale mapping of chromosomal positions of high and low transcription propensity in E. coli using barcoded reporters [75], in which it was revealed that transcriptional level has negative correlation with AT content fraction.

The average transcription level of MucR1 target genes is generally higher than that of non-target genes under both free- 

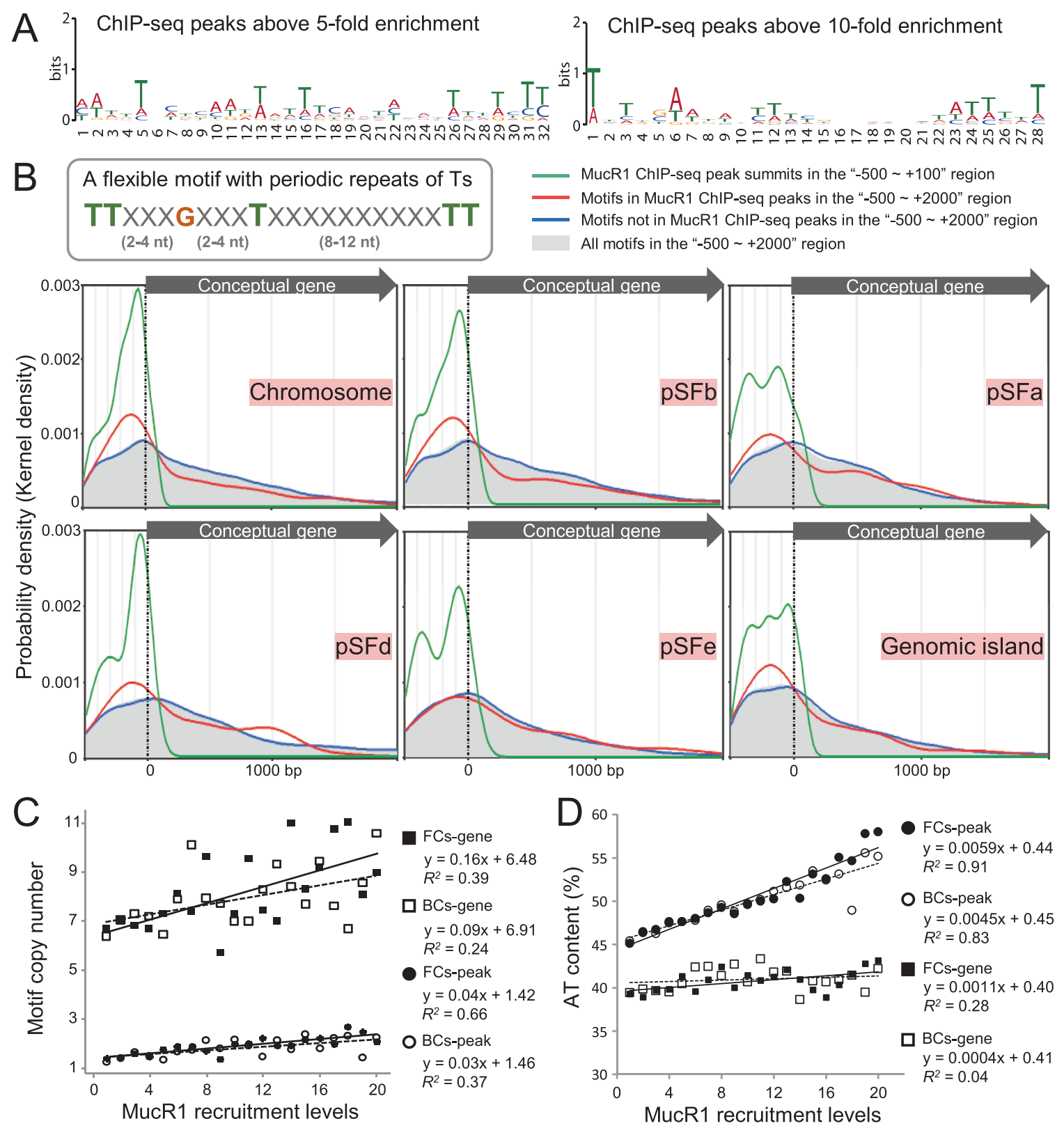

Fig. 6 MucR1 targets are enriched in AT-rich sequences. A In silico deduced MucR1-binding motif within the 200 bp central region of ChIPseq peaks with different enrichment folds compared to the input DNA. B Biased distribution of a flexible motif with periodic repeats of Ts in promoter regions across replicons and genomic islands (the black arrow indicates that only motifs within coding regions were considered in the " $+1 \sim+2000$ " region to avoid interference by downstream intergenic sequences of those genes shorter than $2000 \mathrm{bp}$ ). Distribution of ChIP-seq peak summits within the " $-500 \sim+100 "$ region is shown for comparison. C Relationship between the number of the flexible motif (B) in individual ChIP-seq peaks (within the $200 \mathrm{bp}$ central region; filled and open circles) or peak-associated genes (filled and open boxes) and MucR1 recruitment levels in the corresponding ChIP-seq peaks (horizontal axis). D Relationship between AT\% of individual peaks (within the $200 \mathrm{bp}$ central region; filled and open boxes) or peak-associated genes (filled and open boxes) and MucR1 recruitment levels in the corresponding ChIP-seq peaks (horizontal axis). FCs free-living ChIP-seq, BCs bacteroid ChIP-seq.

living (TY medium; $\mathrm{OD}_{600}=0.5,1.2$, and 4.2) and symbiotic (bacteroids from nodules of cultivated soybeans JD17 and C08, and wild soybean W05) conditions within each conservation levels (Fig. 5A) and replicons (Supplementary Fig. S4A). In the mucR1 mutant, average transcription level of MucR1 target genes is higher than in the wild-type SF45436 (Fig. 5B and Supplementary Fig. S4B), particularly for those target genes (free-living, 144; bacteroids, 170) significantly differentially expressed (DEGs) between two strains (Fig. 5C and Supplementary Fig. S4C). It is intuitively striking that MucR1 target genes are generally transcribed higher than the non-target genes in the wild-type strain (Fig. 5A). Recently, it has been demonstrated that foreign genes are characterized with a higher AT content in their spacers between -10 and -35 elements than canonical promoters, which enhances the interaction of RNA polymerase with this DNA region and leads to a higher gene transcription level than those controlled by canonical promoters [76]. In line with this view, AT content of spacer sequences between -10 and -35 elements associated with MucR1 target genes is significantly higher than non-target ones regardless of whether predicted spacers are intergenic or intragenic (Fig. 5D). This phenomenon is sigma factor (RpoD, RpoE2, RpoH1, RpoH2 and RpoN) independent (Fig. 5D). 

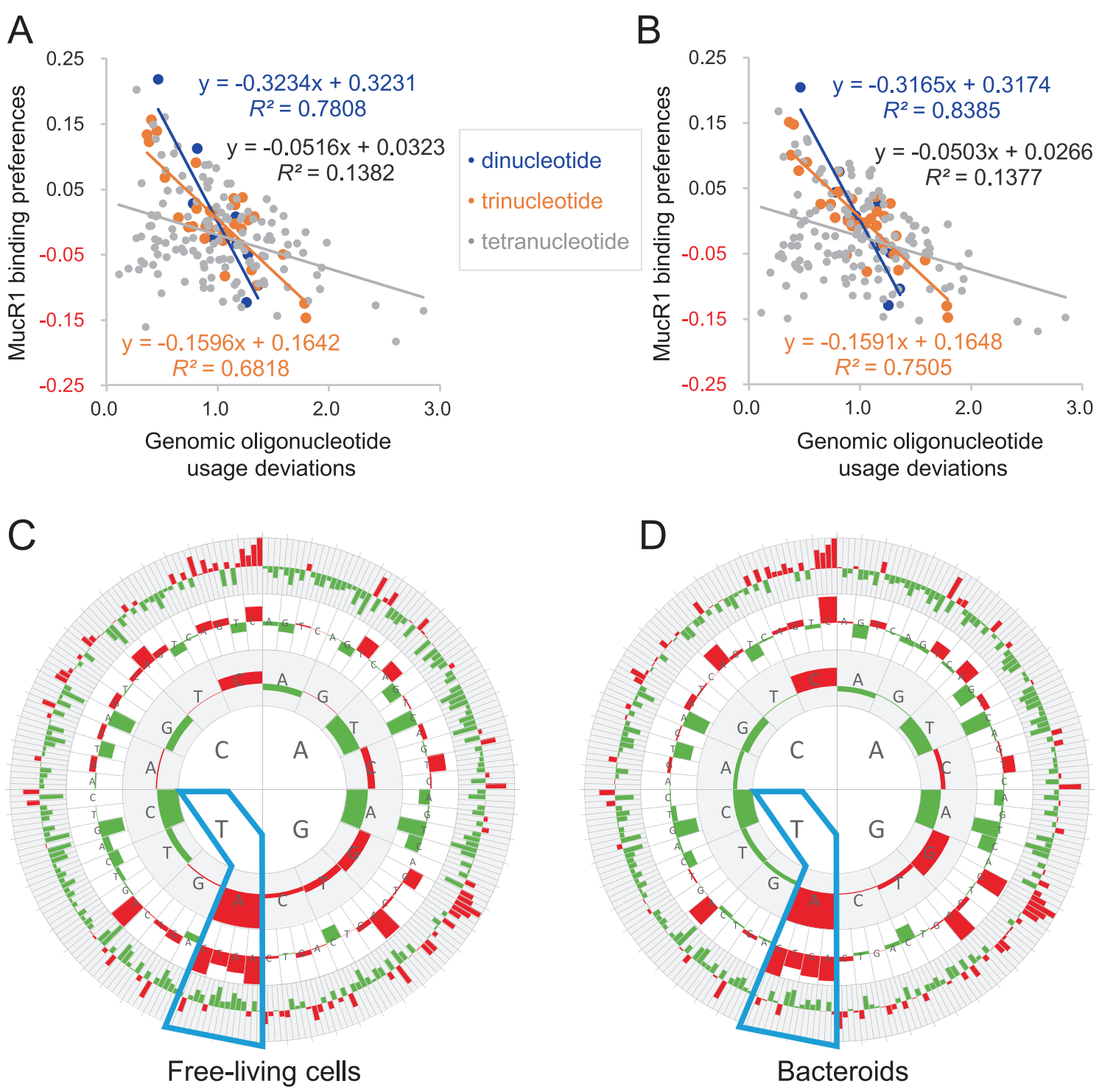

Fig. 7 MucR1 ChIP-seq peaks are enriched with TpA steps. A, B Relation between whole genome usage deviations and MucR1 binding preferences of oligonucleotide under free-living/bacteroid conditions. The height of red/green bars represents the positive/negative coefficient (Spearman's rho) between the oligonucleotide usage deviations and MucR1 recruitment levels in ChIP-seq peaks identified in freeliving cells (C) or bacteroids (D). Bar scales for free-living cells/bacteroids: $-0.129 \sim 0.205 /-0.123 \sim 0.218$ (dinucleotide), $-0.148 \sim 0.151$ / $-0.147 \sim 0.156$ (trinucleotide), $-0.168 \sim 0.168 /-0.182 \sim 0.203$ (tetranucleotide). Oligonucleotide compositions of the $200 \mathrm{bp}$ central regions of ChIP-seq peaks were analyzed.

Therefore, MucR1 has been recruited to down regulate AT-rich foreign genes which are predisposed to be highly transcribed (Fig. 5B-D), though this does not mean a complete repression (Fig. 5A).

\section{MucR1 recruitment levels increase with the number of periodic repeats of Ts and AT content}

Structure of bacterial DNA impacts multiple cellular processes including binding mechanisms of various transcriptional factors such as the intensively studied xenogeneic silencers $[56,77]$. AT-rich DNA is characterized by its more electronegative minor groove than GC-rich DNA, resulting higher affinity of AT-rich DNA for xenogeneic silencers $\mathrm{H}-\mathrm{NS} / \mathrm{Lsr}$ /MvaT with positively charged residues (usually lysine and arginine) [36]. As to MucR/Ros, four basic regions individually harboring two to three arginine/lysine residues are essential for DNA binding [78], and MucR2 carrying a frameshift mutation in one of the basic region is not functional in S. fredii [31]. An in silico motif scanning analysis revealed that the putative MucR1 motifs are fairly degenerate and vary when subsets of peaks with different MucR1 recruitment levels (enrichment folds) were used (Fig. 6A). These flexible patterns have a property of $10-11 \mathrm{bp}$ periodic repeats of $T$ or TT (Fig. 6A), which have been defined as the class $A$ flexible patterns and hypothesized as a putative new category of protein-DNA interaction sites [48]. The structure of the DNA-binding domain of Ros (Ros87) has been obtained [33], to which MucR1 shows $83 \%$ identity in the same domain and shares all conserved key residues involved in DNA binding [9]. Recently, a Ros87-DNA docking model and EMSA demonstrated that two Ts with $11 \mathrm{bp}$ interval and an internal $G$ are required for high affinity interaction between Ros87 and DNA [49]. Interestingly, the high affinity sequence targeted by Ros87 [49] shares the same signature as the typical class A flexible patterns [48] which are shown in Fig. 6B (TTXXXGXXXTXXXXXXXXXXTT). Genome wide analysis revealed that a higher probability density of this flexible motif (Fig. 6B) can be found in MucR1 ChIP-seq peaks than the other regions across different replicons and genomic islands (Fig. 6B). A significant positive 


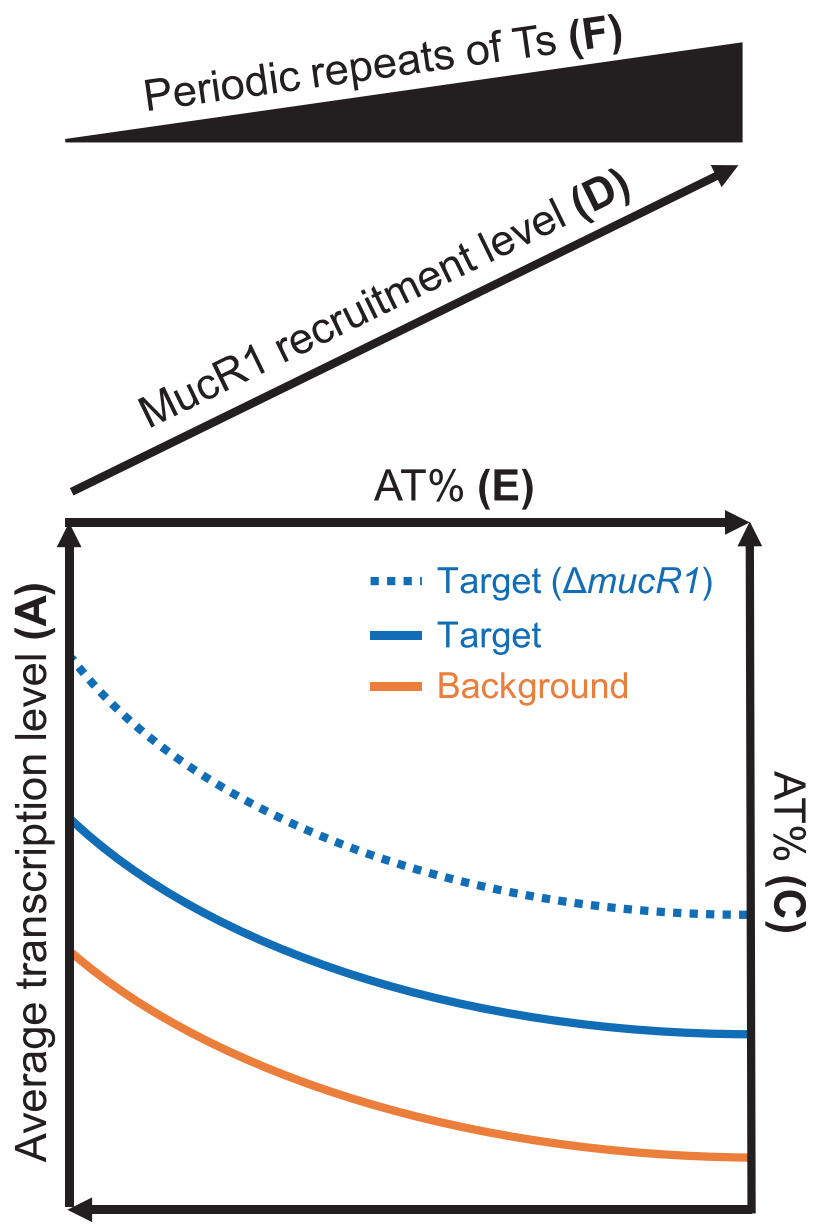

Conservation level (B)

II

III

IV proportion of genus core among target genes negatively correlated with MucR1 recruitment levels of associated peaks (Supplementary Fig. S3B), implying that the more conserved a gene is the less likely being strongly targeted by MucR1.

Similar to other xenogeneic silencers, a positive correlation between AT\% and the protein recruitment level was also found for MucR1 (Fig. 6D; Pearson' rho is 0.96 and 0.93 for free-living and bacteroid ChIP-seq data, respectively; $P$ values $<0.001$ ). These findings imply an intriguing model in which the recruitment levels of MucR1 may gradually decay with the decrease of AT content and the erosion of the typical flexible patterns harboring periodic repeated Ts. This model fits well with the view that AT-rich signature of foreign DNA fragments will be progressively erased in the longterm evolution of bacterial genomes [70], and may allow subsequent selection of cells with the optimal expression levels of foreign genes, given adaptive benefit was explored in particular niches.

The highest-affinity sequences for known convergently evolved xenogeneic silencers H-NS, MvaT, Lsr2 and Rok harbor TpA dinucleotides (often referred as "steps") $[16,80]$. This phenomenon can be partially explained by the fact that TpA steps, among all dinucleotide steps, confer the most flexibility on DNA, facilitating accommodation of these xenogeneic silencers in the minor groove [36]. To determine the potential relationship between oligonucleotide composition of ChIP-seq peaks (tetranucleotide, trinucleotide and dinucleotide) and MucR1 recruitment levels (enrichment fold), Spearman's rho coefficient was calculated. The strongest correlation (rho $=0.389$ and $0.424, P$ value $=1.0 \mathrm{E}-54$ and $8.3 \mathrm{E}-48$ for $\mathrm{FC}$ and $\mathrm{BC}$ peaks, respectively) was found between MucR1 recruitment levels and oligonucleotides composed of $\mathrm{A}$ and $\mathrm{T}$. Considering that the inherent bias in genome sequence might have impact on this analysis, oligonucleotide deviation values [52] instead of content values were further analyzed (Fig. 7A, B). A strong linear relationship between the recruitment level and dinucleotide $\left(R^{2}=0.78\right.$ for FC; $R^{2}=0.84$ for $\mathrm{BC}$ ) or trinucleotide $\left(R^{2}=0.68\right.$ for $\mathrm{FC} ; R^{2}=0.75$ for $\left.\mathrm{BC}\right)$ was observed for both FC (Fig. 7A) and BC (Fig. 7B) samples. Moreover dinucleotide TpA (TA step) and trinucleotides containing TpA had the highest positive correlation with the recruitment level (Fig. 7C, D), though the correlation seems to be weak (rho $=0.205$ and 0.218 , $P$ value $=1.8 \mathrm{E}-15$ and $5.9 \mathrm{E}-13$ for $\mathrm{FC}$ and $\mathrm{BC}$ peaks, respectively).

\section{CONCLUSION}

Horizontal gene transfer (HGT) plays a critical role in increasing bacterial genetic diversity and the ability of exploring previously inaccessible niches $[5,70,81]$ while challenging the regulation integrity. Despite several convergently evolved xenogeneic silencers including $\mathrm{H}-\mathrm{NS}, \mathrm{MvaT}, \mathrm{Lsr} 2$ and Rok have been discovered, it remains largely unexplored about the recruitment mechanisms of beneficial foreign genes. By focusing on the facultative microsymbiont $S$. fredii, this work reveals that the more conserved a gene is, the lower its AT content (Fig. 4B) and the higher its average transcription level (Fig. 5A). MucR1, a conserved zinc-finger regulator in a-proteobacteria, can extensively binds ATrich regions across pangenome subsets of different conservation levels and replicons (Figs. 2C and 4A, B). MucR1 targets with known functions in environmental adaptation and symbiosis are enriched in genus and species core genes implying successful integration of these AT-rich genes (Fig. 3), while ChIP-seq peaks of higher MucR1 recruitment levels are associated with less conserved strain-specific genes of higher AT content and lower transcription levels (Figs. 4D and 5A, Supplementary Fig. S3B). Within each conservation levels, MucR1 down regulates its AT-rich targets which are predisposed to be highly transcribed (Fig. 5A) due to their higher AT\% than non-target genes in the spacer sequences between -35 and -10 elements of promoters which enhance transcription efficiency [76] (Fig. 5C, D). A key mechanism facilitating the recruitment of potential beneficial AT-rich foreign 
genes may at least partially involve the erosion of MucR1-binding sites characterized by periodic repeats of Ts (Fig. 6C). In line with the view that AT-rich signature of foreign DNA can be progressively erased during adaptive evolution [8, 70], this work suggests that MucR directly represses AT-rich foreign genes with predisposed high transcription potential while the progressive erosion of its target sites facilitates the integration of beneficial foreign genes into the regulation network. This adaptive regulation mechanism summarized in Fig. 8 can be common for prokaryotes carrying a xenogeneic silencer managing the adaptive pangenome when exploring various niches.

\section{DATA AVAILABILITY}

Raw sequence data from our RNA-seq and ChIP-seq analyses can be accessed via NCBI Sequence Read Archive (PRJNA389250, PRJNA302586 and PRJNA302588).

\section{REFERENCES}

1. Begon M, Townsend CR. ecology: from individuals to ecosystems, Fifth edit. Wiley, Hoboken, New Jersey, USA; 2021.

2. Charlesworth $D$, Barton NH, Charlesworth B. The sources of adaptive variation. Proc R Soc B Biol Sci. 2017;284:20162864.

3. Svensson El, Berger D. The role of mutation bias in adaptive evolution. Trends Ecol Evol. 2019;34:422-34.

4. Brockhurst MA, Harrison E, Hall JPJ, Richards T, McNally A, MacLean C. The ecology and evolution of pangenomes. Curr Biol. 2019;29:R1094-R1103.

5. Golicz AA, Bayer PE, Bhalla PL, Batley J, Edwards D. Pangenomics comes of age: from bacteria to plant and animal applications. Trends Genet. 2020;36:132-45.

6. Lercher MJ, Pa C. Integration of horizontally transferred genes into regulatory interaction networks takes many million years. Mol Biol Evol. 2008;25:559-67.

7. Tian CF, Zhou YJ, Zhang YM, Li QQ, Zhang YZ, Li DF, et al. Comparative genomics of rhizobia nodulating soybean suggests extensive recruitment of lineagespecific genes in adaptations. Proc Natl Acad Sci USA. 2012;109:8629-34.

8. Langille MGl, Hsiao WWL, Brinkman FSL. Detecting genomic islands using bioinformatics approaches. Nat Rev Microbiol. 2010;8:372-82.

9. Jiao J, Tian C-F. Ancestral zinc-finger bearing protein MucR in alpha-proteobacteria: a novel xenogeneic silencer? Comput Struct Biotechnol J. 2020;18:3623-31.

10. Oshima T, Ishikawa S, Kurokawa K, Aiba H, Ogasawara N. Escherichia coli histonelike protein H-NS preferentially binds to horizontally acquired DNA in association with RNA polymerase. DNA Res. 2006;13:141-53.

11. Grainger DC, Hurd D, Goldberg MD, Busby SJW. Association of nucleoid proteins with coding and non-coding segments of the Escherichia coli genome. Nucleic Acids Res. 2006;34:4642-52.

12. Lucchini S, Rowley G, Goldberg MD, Hurd D, Harrison M, Hinton JCD. H-NS mediates the silencing of laterally acquired genes in bacteria. PLoS Pathog. 2006;2:0746-52.

13. Navarre WW, Porwollik S, Wang Y, McClelland M, Rosen H, Libby SJ, et al. Selective silencing of foreign DNA with low GC content by the H-NS protein in. Salmonella Sci. 2006;313:236-8.

14. Castang S, McManus HR, Turner KH, Dove SL. H-NS family members function coordinately in an opportunistic pathogen. Proc Natl Acad Sci USA. 2008;105:18947-52.

15. Gordon BRG, Li Y, Wang L, Sintsova A, van Bakel $H$, Tian S, et al. Lsr2 is a nucleoidassociated protein that targets AT-rich sequences and virulence genes in Mycobacterium tuberculosis. Proc Natl Acad Sci USA. 2010;107:5154-9.

16. Duan B, Ding P, Hughes TR, Navarre WW, Liu J, Xia B. How bacterial xenogeneic silencer rok distinguishes foreign from self DNA in its resident genome. Nucleic Acids Res. 2018;46:10514-29.

17. Romero IG, Ruvinsky I, Gilad Y. Comparative studies of gene expression and the evolution of gene regulation. Nat Rev Genet. 2012;13:505-16.

18. O'Boyle N, Turner NCA, Roe AJ, Connolly JPR. Plastic circuits: regulatory flexibility in fine tuning pathogen success. Trends Microbiol. 2020;28:360-71.

19. Poole P, Ramachandran V, Terpolilli J. Rhizobia: from saprophytes to endosymbionts. Nat Rev Microbiol. 2018;16:291-303.

20. Batut J, Andersson SG, O'Callaghan D. The evolution of chronic infection strategies in the alpha-proteobacteria. Nat Rev Microbiol. 2004;2:933-45.

21. Jiao J, Ni M, Zhang B, Zhang Z, Young JPW, Chan T-F, et al. Coordinated regulation of core and accessory genes in the multipartite genome of Sinorhizobium fredii. PLOS Genet. 2018;14:e1007428.

22. Cui W, Zhang B, Zhao R, Liu L, Jiao J, Zhang Z, et al. Lineage-specific rewiring of core pathways predating innovation of legume nodules shapes symbiotic efficiency. mSystems. 2021;6:1-18.

23. Harrison PW, Lower RPJ, Kim NKD, Young JPW. Introducing the bacterial 'chromid': not a chromosome, not a plasmid. Trends Microbiol. 2010;18:141-8.
24. Bertram-Drogatz PA, Quester I, Becker A, Puhler A. The Sinorhizobium meliloti MucR protein, which is essential for the production of high-molecular-weight succinoglycan exopolysaccharide, binds to short DNA regions upstream of exoH and exoY. Mol Gen Genet. 1998;257:433-41.

25. Bittinger MA, Milner JL, Saville BJ, Handelsman J. rosR, a determinant of nodulation competitiveness in Rhizobium etli. Mol Plant-Microbe Interact. 1997;10:180-6.

26. Bertram-Drogatz PA, Ruberg S, Becker A, Puhler A. The regulatory protein MucR binds to a short DNA region located upstream of the mucR coding region in Rhizobium meliloti. Mol Gen Genet. 1997;254:529-38.

27. Janczarek M, Skorupska A. The Rhizobium leguminosarum bv. trifolii RosR: transcriptional regulator involved in exopolysaccharide production. Mol PlantMicrobe Interact. 2007;20:867-81.

28. Mueller K, Gonzalez JE. Complex regulation of symbiotic functions is coordinated by MucR and quorum sensing in Sinorhizobium meliloti. J Bacteriol. 2011;193:485-96.

29. Bahlawane C, Mclntosh M, Krol E, Becker A. Sinorhizobium meliloti regulator MucR couples exopolysaccharide synthesis and motility. Mol Plant-Microbe Interact. 2008;21:1498-509.

30. Baglivo I, Russo L, Esposito S, Malgieri G, Renda M, Salluzzo A, et al. The structural role of the zinc ion can be dispensable in prokaryotic zinc-finger domains. Proc Natl Acad Sci USA. 2009;106:6933-8.

31. Jiao J, Wu $\sqcup$, Zhang B, Hu Y, Li Y, Zhang XX, et al. MucR is required for transcriptional activation of conserved ion transporters to support nitrogen fixation of Sinorhizobium fredii in soybean nodules. Mol Plant-Microbe Interact. 2016;29:352-61.

32. Schäper S, Wendt H, Bamberger J, Sieber V, Schmid J, Becker A. A bifunctional UDP-Sugar 4-epimerase supports biosynthesis of multiple cell surface polysaccharides in Sinorhizobium meliloti. J Bacteriol. 2019;201:e00801-18.

33. Malgieri G, Russo L, Esposito S, Baglivo I, Zaccaro L, Pedone EM, et al. The prokaryotic Cys2His2 zinc-finger adopts a novel fold as revealed by the NMR structure of Agrobacterium tumefaciens Ros DNA-binding domain. Proc Natl Acad Sci USA. 2007;104:17341-6.

34. Pirone L, Pitzer JE, D'Abrosca G, Fattorusso R, Malgieri G, Pedone EM, et al. Identifying the region responsible for Brucella abortus MucR higher-order oligomer formation and examining its role in gene regulation. Sci Rep. 2018;8:17238.

35. Baglivo I, Pirone L, Malgieri G, Fattorusso R, Roop RM, Pedone EM, et al. MucR binds multiple target sites in the promoter of its own gene and is a heat-stable protein: Is MucR a H-NS-like protein? FEBS Open Biol. 2018;8:711-8.

36. Singh K, Milstein JN, Navarre WW. Xenogeneic silencing and its impact on bacterial genomes. Annu Rev Microbiol. 2016;70:199-213.

37. Fumeaux C, Radhakrishnan SK, Ardissone S, Théraulaz L, Frandi A, Martins D, et al. Cell cycle transition from S-phase to G1 in Caulobacter is mediated by ancestral virulence regulators. Nat Commun. 2014;5:4081.

38. Li YZ, Wang D, Feng XY, Jiao J, Chen WX, Tian CF. Genetic analysis reveals the essential role of nitrogen phosphotransferase system components in Sinorhizobium fredii CCBAU 45436 symbioses with soybean and pigeonpea plants. Appl Environ Microbiol. 2016;82:1305-15.

39. Liu LX, Li QQ, Zhang YZ, Hu Y, Jiao J, Guo HJ, et al. The nitrate-reduction gene cluster components exert lineage-dependent contributions to optimization of Sinorhizobium symbiosis with soybeans. Environ Microbiol. 2017;19:4926-38.

40. Vincent JM. A manual for the practical study of root nodule bacteria. Blackwell: Oxford; 1970.

41. Fu L, Niu B, Zhu Z, Wu S, Li W. CD-HIT: accelerated for clustering the next generation sequencing data. Bioinformatics. 2012;28:3150-2.

42. Wu S, Zhu Z, Fu L, Niu B, Li W. WebMGA: a customizable web server for fast metagenomic sequence analysis. BMC Genomics. 2011;12:444.

43. Kovach ME, Elzer PH, Hill DS, Robertson GT, Farris MA, Roop RM 2nd, et al. Four new derivatives of the broad-host-range cloning vector pBBR1MCS, carrying different antibiotic-resistance cassettes. Gene. 1995;166:175-6.

44. Quandt J, Hynes MF. Versatile suicide vectors which allow direct selection for gene replacement in Gram-negative bacteria. Gene. 1993;127:15-21.

45. Zhang P, Zhang B, Jiao J, Dai S-Q, Chen W-X, Tian C-F. Modulation of symbiotic compatibility by rhizobial zinc starvation machinery. MBio. 2020;11:e03193-19.

46. Zhang Y, Liu T, Meyer CA, Eeckhoute J, Johnson DS, Bernstein BE, et al. Modelbased analysis of ChIP-Seq (MACS). Genome Biol. 2008;9:R137.

47. Yu Y, Ouyang Y, Yao W. ShinyCircos: An R/Shiny application for interactive creation of Circos plot. Bioinformatics. 2018;34:1229-31.

48. Larsabal E, Danchin A. Genomes are covered with ubiquitous $11 \mathrm{bp}$ periodic patterns, the 'class A flexible patterns'. BMC Bioinformatics. 2005;6:206.

49. Russo L, Palmieri M, Caso JV, Abrosca GD, Diana D, Malgieri G, et al. Towards understanding the molecular recognition process in prokaryotic zinc-finger domain. Eur J Med Chem. 2015;91:100-8.

50. Bertelli C, Laird MR, Williams KP, Lau BY, Hoad G, Winsor GL, et al. IslandViewer 4: expanded prediction of genomic islands for larger-scale datasets. Nucleic Acids Res. 2017;45:W30-W35.

51. Wickham H. Ggplot2. Wiley Interdiscip Rev Comput Stat. 2011;3:180-5. 
52. Pride DT, Meinersmann RJ, Wassenaar TM, Blaser MJ. Evolutionary implications of microbial genome tetranucleotide frequency biases. Genome Res. 2003;13:145-56.

53. Schlüter J, Reinkensmeier J, Barnett MJ, Lang C, Krol E, Giegerich R, et al. Global mapping of transcription start sites and promoter motifs in the symbiotic aproteobacterium Sinorhizobium meliloti 1021. BMC Genomcis. 2013;14:156.

54. Beckstette M, Homann R, Giegerich R, Kurtz S. Fast index based algorithms and software for matching position specific scoring matrices. BMC Bioinforma. 2006;7:1-25.

55. Caswell CC, Elhassanny AEM, Planchin EE, Roux CM, Weeks-Gorospe JN, Ficht TA, et al. Diverse genetic regulon of the virulence-associated transcriptional regulator MucR in Brucella abortus 2308. Infect Immun. 2013;81:1040-51.

56. Dame RT, Rashid FZM, Grainger DC. Chromosome organization in bacteria: mechanistic insights into genome structure and function. Nat Rev Genet. 2020;21:227-42.

57. Sauviac L, Philippe H, Phok K, Bruand C. An extracytoplasmic function sigma factor acts as a general stress response regulator in Sinorhizobium meliloti. J Bacteriol. 2007;189:4204-16.

58. Bastiat B, Sauviac L, Bruand C. Dual control of Sinorhizobium meliloti RpoE2 sigma factor activity by two PhyR-type two-component response regulators. J Bacteriol. 2010;192:2255-65.

59. Sourjik V, Muschler P, Scharf B, Schmitt R. VisN and VisR are global regulators of chemotaxis, flagellar, and motility genes in Sinorhizobium (Rhizobium) meliloti. J Bacteriol. 2000;182:782-8.

60. Scharf B, Rotter C, Muhlbacher S, Salamon D, Schmitt R. Rem, a new transcriptional activator of motility and chemotaxis in Sinorhizobium meliloti. J Bacteriol. 2006;188:6932-42.

61. Machado D, Pueppke SG, Vinardel JM, Ruiz-Sainz JE, Krishnan HB. Expression of nodD1 and nodD2 in Sinorhizobium fredii, a nitrogen-fixing symbiont of soybean and other legumes. Mol Plant-Microbe Interact. 1998;11:375-82.

62. Fellay R, Hanin M, Montorzi G, Frey J, Freiberg C, Golinowski W, et al. nodD2 of Rhizobium sp. NGR234 is involved in the repression of the nodABC operon. Mol Microbiol. 1998;27:1039-50.

63. Zhao R, Liu LX, Zhang YZ, Jiao J, Cui WJ, Zhang B, et al. Adaptive evolution of rhizobial symbiotic compatibility mediated by co-evolved insertion sequences. ISME J. 2018;12:101-11.

64. Rehman HM, Cheung W-L, Wong K-S, Xie M, Luk C-Y, Wong F-L, et al. High-throughput mass spectrometric analysis of the whole proteome and secretome from Sinorhizobium fredii strains CCBAU25509 and CCBAU45436. Front Microbiol. 2019;10:2569.

65. Yang S, Tang F, Gao M, Krishnan HB, Zhu H. R gene-controlled host specificity in the legume-rhizobia symbiosis. Proc Natl Acad Sci USA. 2010;107:18735-40.

66. Geiger O, Röhrs V, Weissenmayer B, Finan TM, Thomas-Oates JE. The regulator gene $p h o B$ mediates phosphate stress-controlled synthesis of the membrane lipid diacylglyceryl-N,N,N-trimethylhomoserine in Rhizobium (Sinorhizobium) meliloti. Mol Microbiol. 1999;32:63-73.

67. diCenzo GC, Sharthiya H, Nanda A, Zamani M, Finan TM. PhoU allows rapid adaptation to high phosphate concentrations by modulating PstSCAB transport rate in Sinorhizobium meliloti. J Bacteriol. 2017;199:e00143-17.

68. Crespo-Rivas JC, Navarro-Gómez P, Alias-Villegas C, Shi J, Zhen T, Niu Y, et al. Sinorhizobium fredii $\mathrm{HH} 103$ RirA is required for oxidative stress resistance and efficient symbiosis with soybean. Int J Mol Sci. 2019;20:787.

69. Schäper S, Steinchen W, Krol E, Altegoer F, Skotnicka D, Søgaard-Andersen L, et al. AraC-like transcriptional activator CuxR binds c-di-GMP by a PilZ-like mechanism to regulate extracellular polysaccharide production. Proc Natl Acad Sci USA. 2017;114:E4822-E4831.

70. Doolittle WF, Nesbo $\mathrm{CL}$, Bapteste E, Zhaxybayeva O. Lateral gene transfer. In: Pagel M, Pomiankowski A (eds). Evolutionary genomics and proteomics. Sinauer Associates Inc.: Sunderland; 2008, p. 45-79.

71. Rocha EPC, Danchin A. Base composition bias might result from competition for metabolic resources. Trends Genet. 2002;18:291-4.

72. Dietel AK, Merker $H$, Kaltenpoth $M$, Kost C. Selective advantages favour high genomic AT-contents in intracellular elements. PLoS Genet. 2019;15:e1007778.

73. Hershberg R, Petrov DA. Evidence that mutation is universally biased towards AT in bacteria. PLoS Genet. 2010;6:e1001115.

74. Raghavan R, Kelkar YD, Ochman H. A selective force favoring increased $\mathrm{G}+\mathrm{C}$ content in bacterial genes. Proc Natl Acad Sci USA. 2012;109:14504-7.

75. Scholz SA, Diao R, Wolfe MB, Fivenson EM, Lin XN, Freddolino PL. High-resolution mapping of the Escherichia coli chromosome reveals positions of high and low transcription. Cell Syst. 2019;8:212-225.e9.
76. Warman EA, Singh SS, Gubieda AG, Grainger DC. A non-canonical promoter element drives spurious transcription of horizontally acquired bacterial genes. Nucleic Acids Res. 2020;48:4891-901.

77. Dorman CJ, Schumacher MA, Bush MJ, Brennan RG, Buttner MJ. When is a transcription factor a NAP? Curr Opin Microbiol. 2020;55:26-33.

78. Esposito S, Baglivo I, Malgieri G, Russo L, Zaccaro L, D'Andrea LD, et al. A nove type of zinc finger DNA binding domain in the Agrobacterium tumefaciens transcriptional regulator Ros. Biochemistry. 2006;45:10394-405.

79. Yousuf M, luliani I, Veetil RT, Seshasayee ASN, Sclavi B, Lagomarsino, MC et al Early fate of exogenous promoters in E. coli. Nucleic Acids Res. 2020;48:2348-56.

80. Ding P, McFarland KA, Jin S, Tong G, Duan B, Yang A, et al. A novel AT-rich DNA recognition mechanism for bacterial xenogeneic silencer MvaT. PLoS Pathog. 2015;11:e1004967.

81. Mclnerney JO, McNally A, O'Connell MJ. Why prokaryotes have pangenomes. Nat Microbiol. 2017:2:17040.

\section{ACKNOWLEDGEMENTS}

This work was supported by the National Key Research and Development Program of China (grant number 2019YFA09004700), National Natural Science Foundation of China (grant number 32070078), the Innovative Project of State Key Laboratory of Agrobiotechnology (grant numbers 2020SKLAB1-5) and the 2115 Talent Development Program of China Agricultural University.

\section{AUTHOR CONTRIBUTIONS}

C-FT and ZZ conceived and designed the research. JJ and M-LL performed experiments. JJ, BZ, and C-FT analyzed and visualized data. C-FT, JJ, BZ, M-LL, and $\mathrm{ZZ}$ prepared the manuscript. C-FT reviewed and edited the manuscript. All authors approved the final version.

\section{COMPETING INTERESTS}

The authors declare no competing interests.

\section{ADDITIONAL INFORMATION}

Supplementary information The online version contains supplementary material available at https://doi.org/10.1038/s41396-021-01118-2.

Correspondence and requests for materials should be addressed to Ziding Zhang or Chang-Fu Tian.

Reprints and permission information is available at http://www.nature.com/ reprints

Publisher's note Springer Nature remains neutral with regard to jurisdictional claims in published maps and institutional affiliations.

\footnotetext{
Open Access This article is licensed under a Creative Commons Attribution 4.0 International License, which permits use, sharing, adaptation, distribution and reproduction in any medium or format, as long as you give appropriate credit to the original author(s) and the source, provide a link to the Creative Commons license, and indicate if changes were made. The images or other third party material in this article are included in the article's Creative Commons license, unless indicated otherwise in a credit line to the material. If material is not included in the article's Creative Commons license and your intended use is not permitted by statutory regulation or exceeds the permitted use, you will need to obtain permission directly from the copyright holder. To view a copy of this license, visit http://creativecommons. org/licenses/by/4.0/.
}

(c) The Author(s) 2021 